Results For the highest compared with the lowest quartile of intake, total omega 3 dietary intake $(\mathrm{OR}=0.68,95 \%$ CI 0.40 to 1.15 , $\mathrm{p}=0.14)$, DHA (OR=0.71, 95\% CI 0.48 to $1.05, \mathrm{p}=0.09)$ and EPA $(\mathrm{OR}=0.72,95 \% \mathrm{CI} 0.46$ to $1.14, \mathrm{p}=0.16)$, all showed non-significant trends with decreased $\mathrm{PrCa}$ risk. However, increased supplement dosage of DHA and EPA both showed significant, protective doseresponse relationships ( $\mathrm{p}$ for trends $=0.04$ ).

Conclusion This study of early onset prostate cancer has shown protective trends for supplement intakes of DHA and EPA which if confirmed in other studies could have implications for prevention.

\section{P2-251 INDUSTRIAL POLLUTION AND CANCER IN SPAIN; A SIMPLE INDUSTRIALISATION INDEX}

doi:10.1136/jech.2011.142976j.84

\author{
${ }^{1,2}{ }^{R}$ Ramis, ${ }^{*} \mathrm{P}$ Diggle, ${ }^{1} \mathrm{E}$ Boldo, ${ }^{1} \mathrm{~J}$ Garcia-Perez, ${ }^{1} \mathrm{P}$ Fernandez, ${ }^{1} \mathrm{G}$ Lopez-Abente. \\ ${ }^{1}$ National Centre for Epidemiology, Carlos III Health Institute, Madrid, Spain; ${ }^{2}$ Lancaster \\ University, Lancaster, UK
}

Introduction Our objective is to study the relation between industrial pollution and cancer in Spain by defining a simple industrialisation index (town level), using the information from the European Pollutant Release and Transfer Register (E-PRTR).

Materials and Methods We used data on industries from the PRTRSpain for year 2007. We selected the 3458 facilities with positively validated co-ordinates. For the 8098 Spanish towns we defined the index as a factor with four levels based on the number of factories in a radius of $2.5 \mathrm{~km}$ from the centroid of each town: Value 0 for towns with no factory within the radius; 1 for those with 1 factory; 2 for those with 2, 3 or 4; and 3 for those with more than four factories. The index, along with socio-demographic indicators (potential confounders), was included in a spatial Poisson model (BYM) to estimate the cancer mortality risk associated with the index levels. We fitted models for 33 cancer causes. Also trend tests were computed.

Results Many causes, 19, showed statistical association with at least one level of the factor. Almost all tumours related to the digestive system and the respiratory system showed excess of risk and/or trend linked with the index.

Conclusions The proposed index is a useful tool to explore possible associations between the level of industrialisation of residential areas and its health outcomes, despite of its limitations. Digestive and respiratory system tumours could be associated to residence near to industrial areas.

\section{P2-252 SHORT- AND LONG-TERM MORTALITY OF ABORIGINAL PEOPLE AFTER HOSPITAL ADMISSION FOR ISCHAEMIC HEART DISEASE: A DATA LINKAGE STUDY IN NEW SOUTH WALES, AUSTRALIA}

doi:10.1136/jech.2011.142976j.85

${ }^{1} \mathrm{D}$ Randall, ${ }^{*} 1,2 \mathrm{~L}$ Jorm, ${ }^{3} \mathrm{~A}$ Leyland, ${ }^{1} \mathrm{~S}$ Lujic, ${ }^{2} \mathrm{~T}$ Churches, ${ }^{2} \mathrm{M}$ Haines, ${ }^{4} \mathrm{~S}$ Eades, ${ }^{1}$ A O'Loughlin. ${ }^{1}$ University of Western Sydney, Sydney, New South Wales, Australia;

${ }^{2}$ The Sax Institute, Sydney, New South Wales, Australia; ${ }^{3}$ Social and Public Health Sciences Unit, Glasgow, Scotland, UK; ${ }^{4}$ Baker IDI Heart and Diabetes Institute, Melbourne, Victoria, Australia

Introduction The health of Australian Aboriginal people is worse than that of non-Aboriginal people across every conceivable indicator. Ischaemic heart disease (IHD) is a leading cause of premature death. We aimed to determine whether short- and long-term mortality after hospitalisation for IHD was higher for Aboriginal than non-Aboriginal patients, in order to guide interventions to reduce the mortality gap.
Method New South Wales hospital data (July 2000-December 2008) were linked to mortality data (July 2000-December 2009). The first IHD admission per person was selected from January 2002. We ran multilevel logistic regression models for 30-day and 365-day mortality with 131357 patients clustered within 193 public hospitals.

Results After adjusting for age, sex and a random hospital intercept, Aboriginal IHD patients had similar odds of dying within 30 days as non-Aboriginal patients (AOR: 1.1, 0.9-1.4). Using the same adjustments, odds of dying within 365 days were significantly higher for Aboriginal than non-Aboriginal patients (AOR: 1.4, 1.2-1.7). Adding the Charlson co-morbidity index and specific IHD diagnosis reduced this 365-day AOR to 1.2 (1.0-1.4). After adding the above variables, $2.4 \%$ of the unexplained variation in 365 -day mortality was attributable to hospitals.

Conclusion Aboriginal IHD patients in NSW had similar odds of dying within 30 days of admission as non-Aboriginal patients, but were more likely to die within 1 year of admission. Increased longterm mortality was partly explained by individual risk factors. Tackling the mortality gap will require major efforts to boost primary and secondary prevention, as well as improving hospital care for IHD.

\section{P2-253 ALCOHOL MISUSE AMONG PARTNERS: A POTENTIAL EFFECT MODIFIER IN THE RELATIONSHIP BETWEEN PHYSICAL INTIMATE PARTNER VIOLENCE AND POSTPARTUM DEPRESSION}

doi:10.1136/jech.2011.142976j.86

${ }^{1} \mathrm{M}$ Reichenheim, ${ }^{*}{ }^{2} \mathrm{G}$ Lobato, ${ }^{3} \mathrm{~A}$ D Oliveira, ${ }^{1,4} \mathrm{C}$ L Moraes. ${ }^{1}$ Department of Epidemiology, Institute of Social Medicine (IMS), Rio de Janeiro State University (UERJ), Rio de Janeiro, Brazil; ${ }^{2}$ Fernandes Figueira Institute, Oswaldo Cruz Foundation (FIOCRUZ), Rio de Janeiro, Brazil; ${ }^{3}$ Bezerra de Araújo Faculty (FABA), Rio de Janeiro, Brazil; ${ }^{4}$ Family Health Program, Estácio de Sá University, Rio de Janeiro, Brazil

Introduction This study evaluated if the probability of postpartum depression (PPD) increases with an upward gradient of physical intimate partner violence (IPV) during pregnancy and whether substance use by any member of the couple modifies this relationship.

Methods The sample comprised 811 randomly selected mothers of children under five months old attending primary health services of Rio de Janeiro, Brazil. The Revised Conflict Tactics Scale (CTS2) gauged physical IPV, and the Edinburgh Postnatal Depression Scale (EPDS) assessed PPD. A hierarchical logistic regression model was employed to deal with confounding. Specific interaction terms between physical IPV and alcohol misuse or use of illicit drugs were also tested.

Results Physical IPV during pregnancy was reported by $37.8 \%$ of respondents and $24.3 \%$ were presumably depressed (EPDS score $\geq 12$ ). Interaction between physical IPV and partners' alcohol misuse was statistically significant ( $p$ value $=0.026$ ). Although there was a significant rise of PPD with just one act of physical IPV in the absence of a partners' alcohol misuse, mounting acts did not have any further influence. Conversely, when partners misused alcohol, the probability progressively and steeply increased from two acts onwards, reaching almost sevenfold by six cumulative physical IPV events as opposed to none.

Discussion Results reinforce the relevance of physical IPV as a risk factor to PPD. They also suggest that context matters, partners' alcohol misuse acting as an important effect modifier. These evidences justify tailored preventive, screening and intervention procedures for IPV and alcohol misuse during pregnancy and the postpartum period. 